\title{
Mapping evidence of free maternal healthcare financing and quality of care in sub-Saharan Africa: a systematic scoping review protocol
}

\author{
Monica Ansu-Mensah¹, Tahiru Mohammed ${ }^{1}$, Roseline H. Udoh¹, Vitalis Bawontuo ${ }^{1,3}$ and Desmond Kuupiel ${ }^{2,3^{*}}$ (B)
}

\begin{abstract}
Background: Identifying and addressing research gaps on the implementation of free maternal healthcare financing policies and the quality of service delivery in sub-Saharan Africa (SSA) is essential in ensuring continuous patronage of the services by clients and sustainability of the policies to meet the intended goals. The proposed scoping review is aimed at mapping evidence on free maternal healthcare financing and quality of care in SSA.

Methods: Arksey and O'Malley's framework, Levac and colleagues' recommendations, and the Joanna Briggs Institute guidelines will be used to guide the proposed study. A complete keyword search for relevant studies presenting evidence on free maternal healthcare financing and perceived quality of care among women in SSA will be performed in EBSCOhost, PubMed, Web of Science, Google Scholar and SCOPUS. Relevant grey literature from university repositories and international organisations such as WHO and government websites, and the reference lists of included studies will be additionally searched. The Preferred Reporting Items for Systematic Reviews and Meta-analysis: Extension for Scoping Review (PRISMA-ScR) will be used to present the results of the proposed scoping review. NVivo version 11 software package will be employed to extract the relevant outcomes from the included studies using content thematic analysis. Quality appraisal of the included studies for this proposed study will be performed utilising the latest mixed methods appraisal tool.
\end{abstract}

Discussion: It is anticipated that the results of the proposed study will inform future research and reveal evidencebased information to address potential quality of care issues that may arise because of free maternal healthcare policy implementation in some SSA countries. The proposed study will also be useful to other SSA countries planning to implement free maternal health policy, as they will be able to draw useful lessons to guide them through the process.

Keywords: Maternal healthcare, health financing, free maternal healthcare, quality of care, sub-Saharan Africa

\section{Background}

Reducing maternal and child mortality remains a global challenge. This challenge received much attention during the launch of the Millennium Development Goals in the year 2000 by the United Nations and partners such as WHO [1-5]. It is estimated that $99 \%$ of all maternalrelated deaths occur in low- and middle-income countries,

\footnotetext{
* Correspondence: desmondkuupiel98@hotmail.com

${ }^{2}$ Research for Sustainable Development Consult, Sunyani, Ghana

${ }^{3}$ Discipline of Public Health Medicine, School of Nursing and Public Health,

University of KwaZulu-Natal, Durban, South Africa

Full list of author information is available at the end of the article
}

of which most (66\%) are located in sub-Saharan Africa (SSA) [5-8]. Maternal mortality in SSA is 1 in 39 live births, whereas it is 1 in 3800 live births in high-income countries [9, 10], 1 in every 130 live births in Oceania, and 1 in every 160 live births Southern Asia [5-8]. Therefore, increasing access to quality maternal healthcare services in SSA has been a global priority since the inception of the Millennium Development Goals to date.

To improve access to maternal healthcare services for all women, particularly the poor, various health financing policies, such as free maternal healthcare schemes,

(c) The Author(s). 2019 Open Access This article is distributed under the terms of the Creative Commons Attribution 4.0 International License (http://creativecommons.org/licenses/by/4.0/), which permits unrestricted use, distribution, and reproduction in any medium, provided you give appropriate credit to the original author(s) and the source, provide a link to the Creative Commons license, and indicate if changes were made. The Creative Commons Public Domain Dedication waiver (http://creativecommons.org/publicdomain/zero/1.0/) applies to the data made available in this article, unless otherwise stated. 
aimed at removing financial barriers have been adopted and implemented by some SSA countries. Most of these free maternal healthcare policies cover the WHO's priority interventions such as antenatal care, delivery and postnatal care, which are recognised by WHO as essential for improving maternal health outcomes [1, 11]. For instance, with the intervention of antenatal care [11], every pregnant woman is supposed to visit the care provider at least four times during pregnancy for services such as health education, counselling, screening and testing, and treatment, where necessary, with the aim of promoting the health status of both mother and child [9].

Although these free maternal healthcare policies have improved access to healthcare, particularly for pregnant women who are in the low-income brackets, the quality of the service delivery is essential to achieve the aim of the policy $[1,9,11]$. The quality of maternal healthcare services rendered at the various health facilities is particularly crucial to facilitating continuous utilisation of the services by clients, thereby ensuring the desired maternal health outcomes [12-16]. Therefore, the proposed scoping review is aimed at mapping evidence on free maternal healthcare financing and quality of care in SSA focusing on clients, healthcare providers or healthcare professionals, and healthcare managers' perspectives. We anticipate that the results of this scoping review will reveal useful evidence-based information and identify gaps for future studies to improve maternal healthcare delivery in SSA. We also anticipate that the study findings may influence policy decisions to improve the quality of care as well as contribute to the achievement of United Nations Sustainable Development Goal 3.1, which stipulates that a maternal mortality of less than 70 maternal deaths per 100,000 live births should be achieved by 2030 [17].

\section{Methods}

Overview

Scoping reviews are considered useful in representing series of literature that exists around a subject of interest and helps to focus the research questions by registering existing research findings and identifying research gaps [4]. A scoping methodology is also considered a useful approach for determining the basic and value of followup of a primary study or a full systematic review [5]. Based on this, the current scoping review will be guided by the enhanced version of Arksey and O'Malley's framework [18], Levac et al.'s recommendations [19] and the 2015 Joanna Briggs Institute guidelines [20] as well as the Preferred Reporting Items for Systematic Reviews and Meta-Analyses Protocol (PRISMA-P) (Additional file 1). The framework involves the following: identifying the research question; identifying relevant studies; study selection; charting the data; and collating, summarising and reporting results [18].

\section{Identifying the research question}

Our research question is: What is the evidence on free maternal healthcare financing and quality of maternal healthcare in SSA?

The sub-review questions will be as follows:

- What is the perspective of clients on the quality of care provided under the free maternal healthcare policy in SSA?

- What is the perspective of healthcare providers on the quality of care provided under the free maternal healthcare policy in SSA?

- What is the perspective of healthcare managers on the quality of care provided under the free maternal healthcare policy in SSA?

The Population, Concept, and Context (PCC) mnemonic [20] used to determine the eligibility of the primary research question for the proposed scoping review is shown in Table 1.

\section{Identify relevant studies}

We will conduct a thorough and complete search of numerous bibliographic databases to include all relevant studies on free maternal healthcare policies and the quality of healthcare delivery in SSA irrespective of publication standing whether published, unpublished or in press. A full search will be conducted in EBSCOhost, PubMed, Web of Science, Google Scholar and SCOPUS for relevant studies. Relevant grey literature from university repositories and international organisations such as WHO and government websites will also be searched. We will additionally explore the reference lists of all included studies for relevant studies. We will use a combination of the following keywords to search for relevant studies from the electronic databases: 'free maternal healthcare' 'healthcare financing' 'health insurance scheme' 'maternal healthcare' 'quality of care'. We will use Boolean terms 'AND' and 'OR' to separate keywords. We will also include appropriate Medical Subject Heading $(\mathrm{MeSH})$ terms and keywords to identify relevant studies. Study designs will be limited to only qualitative, quantitative or mixed methods studies and the search language limited to English due to a lack of expertise to interpret other languages. However, date of publication limitations will be removed. Table 2 shows a pilot search conducted in PubMed demonstrating the possibility of conducting the proposed scoping review. 
Table 1 PCC (Population, Concept, and Context pneumonic) framework for defining the eligibility of the primary question for the scoping review

\begin{tabular}{ll}
\hline P-Population & $\begin{array}{l}\text { Qualitative, quantitative or mixed methods studies involving free maternal healthcare financing policies: we defined } \\
\text { free maternal healthcare policy as any insurance scheme that seeks to eliminate financial barriers associated with the } \\
\text { use of maternal and child healthcare services by pregnant and post-natal mothers }\end{array}$ \\
C-Concept & $\begin{array}{l}\text { Quality of care: quality of maternal healthcare is defined based on client, healthcare provider and health manager } \\
\text { perspectives }\end{array}$ \\
C-Context & Sub-Saharan Africa: In this study, sub-Saharan Africa will refer to all countries in the WHO African Region [21]
\end{tabular}

\section{Eligibility criteria and study selection}

To ensure the selection of relevant studies for this review, the study selection will be guided by the eligibility criteria as specified under the inclusion/exclusion criteria.

\section{Inclusion criteria}

We will include studies that meet the following criteria:

- Evidence of the study conducted in SSA

- Studies presenting evidence of free maternal healthcare policy

- Studies reporting evidence on quality of maternal healthcare services

- Primary studies

\section{Exclusion criteria}

This study will exclude the following:

- Studies conducted in countries not included in the WHO African Region

- Studies targeting women of fertility age

- Studies reporting evidence on quality of care from the health providers' perspective

- Studies reporting evidence on quality of care from the funders' or representatives of health financing institutions' perspectives

- Other types of reviews

\section{Study selection}

Screening of relevant studies for inclusion in the proposed scoping review will be conducted in three phases. First, the principal investigator will conduct the title screening of retrievable studies from the online database.
All eligible studies will be imported onto an endnote library X7 created for the study. At the second (abstract screening) and third (full-text screening) stages, two reviewers will independently sort the studies into two classifications ('inclusive' and 'exclusive') using the eligibility criteria. Discrepancies between reviewers' responses at the abstract screening stage will be resolved through a discussion by the review team until a consensus is reached. However, discrepancies in reviewers' responses at the full-text screening stage will be resolved by involving a third reviewer. In the situation where a full-text article cannot be found from the databases, assistance would be sought from the Catholic University's library or the full-text will be requested from the authors via email. Inter-rater agreement (Cohen's kappa co-efficient (k) statistics) between reviewers' responses will be calculated along with McNemar's $\chi^{2}$ statistics using Stata 14 following full-text screening. The search record will also be adequately documented as follows: date of search, database, keywords, number of retrievable studies and number of eligible studies. We will follow an adapted PRISMA [21] to present the screening results of the proposed scoping review, as shown in Fig. 1.

\section{Charting the data}

A data charting form will be developed for extraction of relevant data from the included studies in the scoping review. This form will include the following: author and date, study title, objective/aim of the study, study design, study setting, type of free maternal healthcare policy and perceived quality of care. The data extraction form will be piloted by two independent reviewers using a random sample of $10 \%$ of the included studies to ensure consistency and

Table 2 Pilot search in PubMed electronic database

\begin{tabular}{lll}
\hline Date & Database & Keywords \\
\hline 31/08/2019 & PubMed & - Line 1: "free maternal healthcare"[All Fields] OR "free healthcare financing"[MeSH Terms] AND \\
& • Line 2: "mothers"[MeSH Terms] OR "expectant mothers"[All Fields] OR \\
& • Line 3: "pregnant"[All Fields] OR "Pregnancy"[MeSH Terms] OR "post-natal"[All Fields] OR \\
& • Line 4: "women"[All Fields] OR "woman"[All Fields] AND "quality"[All Fields] OR \\
& • Line 5: "quality of health care"[MeSH Terms] OR "quality of maternal healthcare"[All Fields] OR \\
& • Line 6: "quality of care"[All Fields] OR "quality of maternal health service"[All Fields] OR \\
& • "quality of maternal health delivery"[All Fields] AND \\
& • Line 7: "Africa"[All Fields] OR "sub Saharan Africa"[All Fields] \\
& • Line 8: "humans"[MeSH Terms]
\end{tabular}




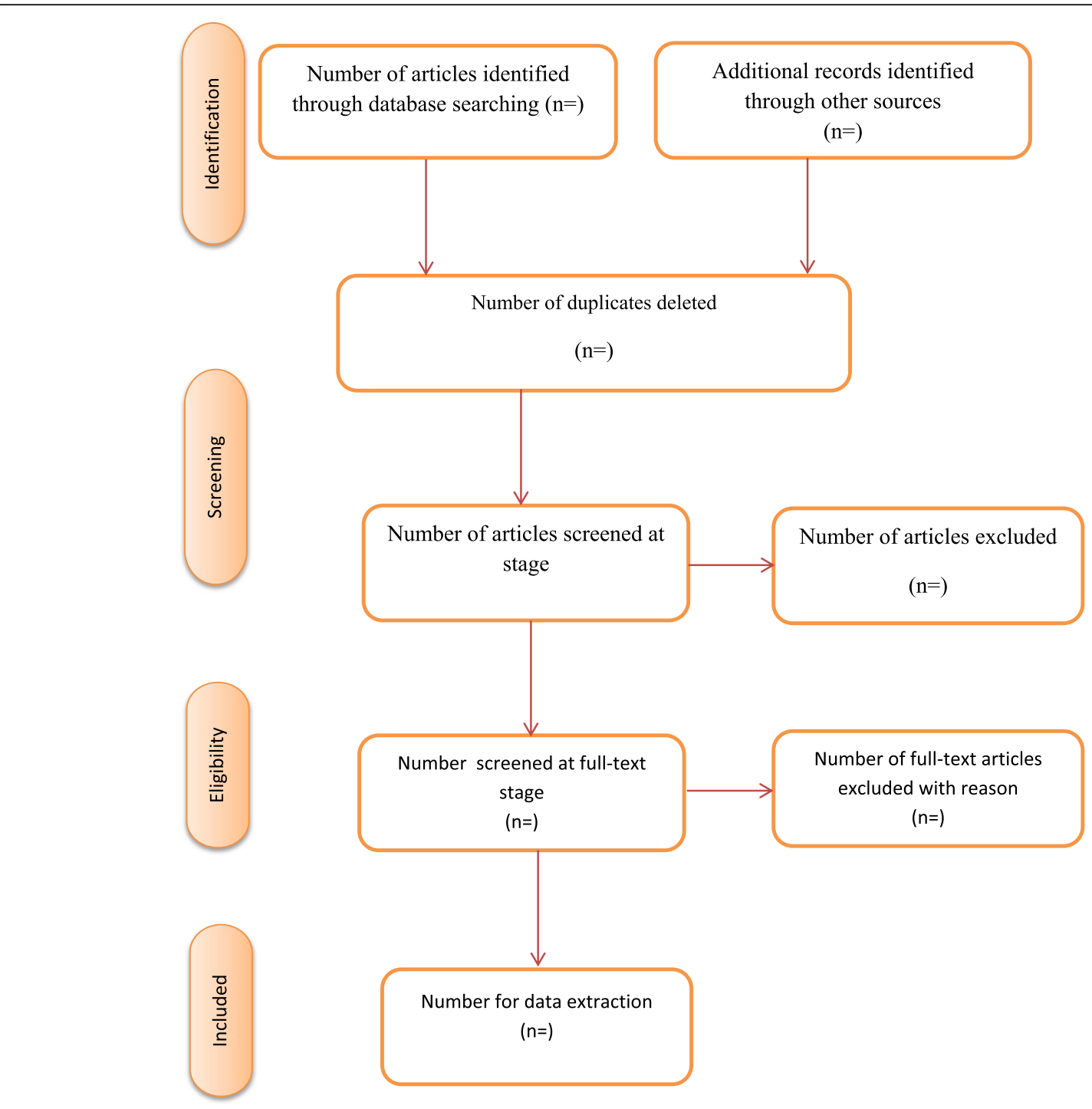

Fig. 1 PRISMA 2009 flow diagram [21]

accuracy. The data extraction form will be adjusted as required based on feedback from the two independent reviewers. We will constantly update the data extraction form to enable adequate abstraction of all relevant data to answer the review question.

\section{Collating, summarising and reporting the results}

We will extract data related to free maternal health policies and perceived quality of care in SSA using content thematic analysis approach [22]. NVivo version 11 will be employed for the content thematic analysis of the included studies and the findings on free maternal health policies and perceived quality of care in SSA summarised and presented using a narrative approach. Emerging themes will also be reported.

\section{Quality appraisal}

To evaluate the quality of the studies that will be included in the proposed scoping review, the Mixed Method Quality Appraisal Tool (MMAT) Version 2018 will be used to evaluate the methodological quality of all the studies included. The relevance of the study, study design, adequacy and methodology, data collection, analysis of data, and study findings will be examined using the MMAT tool. The quality assessment will be helpful in reporting the risk of bias of the studies included. The quality of the included studies will be graded by calculating the total percentage quality score as specified by the 2018 MMAT. A percentage quality score ranging from $\leq 50 \%$ will be considered as low quality, $51-75 \%$ will be considered as average quality, and $76-100 \%$ will be considered as high quality. 


\section{Discussions}

The proposed scoping review will aim to map evidence on free maternal healthcare financing and quality of care in SSA. Improving the quality of care during pregnancy has many benefits such as improved utilisation of antenatal services [23, 24], early detection of infection and prompt linkage to care [25], and reduction of maternal morbidities and mortalities [7], among others. It is anticipated that the results of the proposed study will inform future research and reveal evidence-based information to address the potential quality of care issues that may arise because of free maternal healthcare policy implementation in some SSA countries. The proposed study will also be useful for other SSA countries planning to implement free healthcare financing schemes for maternal health, helping them to draw useful lessons to guide its implementation. The proposed study will thus contribute to healthcare systems strengthening and improve research on free maternal healthcare financing schemes and the quality of care in SSA.

\section{Conclusion}

The results of this systematic scoping review research will help identify gaps useful for future studies, such as systematic reviews, meta-analysis and primary studies, to improve the quality of maternal healthcare delivery services in SSA.

\section{Supplementary information}

Supplementary information accompanies this paper at https://doi.org/10. 1186/s12961-019-0495-1.

Additional file 1. PRISMA-P checklist.

\section{Abbreviations}

MMAT: Mixed Method Quality Appraisal Tool; SSA: sub-Saharan Africa

\section{Acknowledgments}

We owe a debt of gratitude to the Almighty God for seeing us through with this scoping review protocol. We are also thankful to the Catholic University College of Ghana - Fiapre, for providing us with the necessary resources to write this manuscript.

\section{Authors' contribution}

MA-M and DK conceptualised the study. MA-M wrote the protocol. RHU and TM contributed to the writing. DK and VB designed the study methodology and critically reviewed the protocol. MA-M wrote the final draft manuscript and all the authors approved it.

\section{Funding}

No funding has been obtained yet for this study.

\section{Availability of data and materials}

We have duly cited all studies and data is presented in the form of references.

\section{Ethics approval and consent to participate}

Not applicable.

\section{Consent for publication}

Not applicable.

\section{Competing interests}

The authors declare that they have no competing interests.

\section{Author details}

${ }^{1}$ Faculty of Health and Allied Sciences, Catholic University College of Ghana, Fiapre, Sunyani, Ghana. ${ }^{2}$ Research for Sustainable Development Consult, Sunyani, Ghana. ${ }^{3}$ Discipline of Public Health Medicine, School of Nursing and Public Health, University of KwaZulu-Natal, Durban, South Africa.

Received: 8 April 2019 Accepted: 11 October 2019

Published online: 27 November 2019

\section{References}

1. Machira K, Palamuleni M. Women's perspectives on quality of maternal health care services in Malawi. Int J Women's Health. 2018;10:25-34.

2. Travis P, Bennett S, Haines A, Pang T, Bhutta Z, Hyder AA, et al. Overcoming health-systems constraints to achieve the Millennium Development Goals. Lancet. 2004;364(9437):900-6.

3. Sachs JD, MCArthur JW. The millennium project: a plan for meeting the millennium development goals. Lancet. 2005;365(9456):347-53.

4. World Health Organization. Health in 2015: From Millennium Development Goals to Sustainable Development Goals. Geneva: WHO; 2015.

5. Tawiah EO. Maternal health care in five sub-Saharan African countries. J Afr Popul Stud. 2011;25(1). https://doi.org/10.11564/25-1-264.

6. Kassebaum NJ, Bertozzi-Villa A, Coggeshall MS, Shackelford KA, Steiner C, Heuton KR, et al. Global, regional, and national levels and causes of maternal mortality during 1990-2013: a systematic analysis for the Global Burden of Disease Study 2013. Lancet. 2014;384(9947):980-1004.

7. Kuupiel D, Tlou B, Bawontuo V, Mashamba-Thompson TP. Accessibility of pregnancy-related point-of-care diagnostic tests for maternal healthcare in rural primary healthcare facilities in Northern Ghana: a cross-sectional survey. Heliyon. 2019;5(2):e01236.

8. World Health Organization. World Health Statistics 2016: Monitoring Health for the Sustainable Development Goals. Geneva: WHO; 2016.

9. Asante-Sarpong H, Owusu AY, Saravanan S, Appiah E, Abu M. Determinants of use of supervised delivery care under Ghana's fee exemption policy for maternal healthcare: the case of the Central Region. BMC Pregnancy Childbirth. 2016;16:172.

10. Zureick-Brown S, Newby H, Chou D, Mizoguchi N, Say L, Suzuki E, et al. Understanding global trends in maternal mortality. Int Perspect Sex Reprod Health. 2013;39(1). https://doi.org/10.1363/3903213.

11. Arthur E. Wealth and antenatal care use: implications for maternal health care utilisation in Ghana. Heal Econ Rev. 2012;2(1):14.

12. Emelumadu OF, Onyeonoro UU, Ukegbu AU, Ezeama NN, Ifeadike CO, Okezie OK. Perception of quality of maternal healthcare services among women utilising antenatal services in selected primary health facilities in Anambra State, Southeast Nigeria. Niger Med J. 2014;55(2):148.

13. Nnebue C, Ebenebe U, Adinma E, Iyoke C, Obionu C, llika A. Clients' knowledge, perception, and satisfaction with quality of maternal health care services at the primary health care level in Nnewi, Nigeria. Niger J Clin Pract. 2014;17(5):594-601.

14. Rai SK, Dasgupta R, Das M, Singh S, Devi R, Arora N. Determinants of utilization of services under MMJSSA scheme in Jharkhand 'Client Perspective': a qualitative study in a low performing state of India. Indian J Public Health. 2011;55(4):252.

15. Oladapo OT, Iyaniwura CA, Sule-Odu AO. Quality of antenatal services at the primary care level in southwest Nigeria. Afr J Reprod Health. 2008;12(3):71-92.

16. Nair H, Panda R. Quality of maternal healthcare in India: has the National Rural Health Mission made a difference? J Glob Health. 2011; 1(1):79.

17. United Nations. United Nations Sustainable Development Goals 2015. http://www.un.org/sustainabledevelopment/news/communicationsmaterial/. Accessed 6 Apr 2019.

18. Arksey H, O'Malley L. Scoping studies: towards a methodological framework. Int J Soc Res Methodol. 2005;8(1):19-32.

19. Levac D, Colquhoun H, O'Brien KK. Scoping studies: advancing the methodology. Implement Sci. 2010;5:69.

20. Joanna Briggs Institute. Joanna Briggs Institute Reviewers' Manual: 2015 Edition/Supplement. Methodology for JBI Scoping Reviews. Adelaide: The Joanna Briggs Institute; 2015 
21. Moher D, Liberati A, Tetzlaff J, Altman DG. Preferred reporting items for systematic reviews and meta-analyses: the PRISMA statement. Ann Intern Med. 2009;151(4):264-9.

22. Braun V, Clarke V, Hayfield N, Terry G. Thematic analysis. Handbook of Research Methods in Health Social Sciences. Springer; 2019. p. 843-60

23. Ameyaw EK, Kofinti RE, Appiah F. National health insurance subscription and maternal healthcare utilisation across mothers' wealth status in Ghana. Health Econ Rev. 2017;7(1):16.

24. Afulani PA. Determinants of stillbirths in Ghana: does quality of antenatal care matter? BMC Pregnancy Childbirth. 2016;16:132.

25. Majors CE, Smith CA, Natoli ME, Kundrod KA, Richards-Kortum R. Point-ofcare diagnostics to improve maternal and neonatal health in low-resource settings. Lab Chip. 2017;17(20):3351-87.

\section{Publisher's Note}

Springer Nature remains neutral with regard to jurisdictional claims in published maps and institutional affiliations.

Ready to submit your research? Choose BMC and benefit from:

- fast, convenient online submission

- thorough peer review by experienced researchers in your field

- rapid publication on acceptance

- support for research data, including large and complex data types

- gold Open Access which fosters wider collaboration and increased citations

- maximum visibility for your research: over $100 \mathrm{M}$ website views per year

At BMC, research is always in progress.

Learn more biomedcentral.com/submissions 